\title{
Estuezos admisbles de diseño por grado estructural para nueve maderas de plantación de Costa Rica
}

Roger Moya-Roque ${ }^{1}$ Guillermo González Beltrán²

\section{Resumen}

Costa Rica cuenta con madera de varias especies proveniente de plantaciones forestales para abastecer la demanda de madera. Sin embargo, no se tienen los valores de esfuerzos admisibles para grados estructurales de estas maderas. El presente trabajo tiene como objetivo, mostrar los valores de diseño derivados de madera libre de defectos para nueve especies de plantación utilizadas en la reforestación comercial. Los esfuerzos analizados fueron flexión estática, compresión paralela y perpendicular al grano y cortante y tensión paralela al grano. Los resultados en la madera libre de defectos mostró que Tectona grandis, Terminalia oblonga y Acacia mangium presentaron los más altos valores en MOE y MOR en flexión. En compresión paralela y perpendicular, $T$. grandis presentó la más alta resistencia, $A$. mangium y $T$. grandis fueron las especies con la mayor resistencia en tensión paralela al grano. A. mangium y

\begin{abstract}
Allowable stress design by structural grade for nine plantation woods of Costa Rica

Costa Rica has several species wood from forest plantations to supply the demand for end-user wood. However, this country does not have allowable stress design for different structural grade for these woods. This paper aims to show the allowable stress design values derived from clear wood for nine of plantation species used in commercial reforestation in Costa Rica. The mechanical properties analyzed were: static bending, compression parallel and perpendicular to grain and shear and tension parallel to grain. The results in clear wood specimens showed that Tectona grandis, Terminalia oblonga and Acacia mangium presented the highest values of MOE and MOR in static bending. In compression parallel and perpendicular, T. grandis showed the highest resistance. A. mangium and $T$. grandis were the plantation species
\end{abstract}


Cupressus lusitanica mostraron los más altos valores en cortante paralela al grano. Vochysia guatemalensis y Bombacopsis quinata mostraron la resistencia más baja en todas las propiedades medidas. La derivación de los esfuerzos de diseño permitió establecer tres grupos de especies. El grupo 1 está formado por Acacia mangium, $T$. grandis, Terminalia amazonia y $T$. oblonga con los más altos valores admisibles. El grupo 2 , con valores intermedios de valores admisibles, lo componen Gmelina arborea y C. Iusitanica. El grupo 3, con los valores admisibles más bajos, compuesto por Alnus acuminata, $B$. quinata y $V$. guatemalensis.

Palabras clave: Maderas tropicales, plantaciones, crecimiento rápido, grados estructurales, resistencia de la madera, Costa Rica.

\section{Introducción}

La reforestación en Costa Rica surgió como resultado de la implementación de un sistema de incentivos por parte del Estado (Castro et al. 1998), con el objetivo de atenuar el proceso de pérdida de la cobertura boscosa sufrida en las décadas de los 60 s y 70 s del siglo pasado (Sader y Joyce 1988). En este proceso de reforestación se han plantado una gran variedad de especies, tales como melina (Gmelina arborea), teca (Tectona grandis) y acacia (Acacia mangium) entre las especies exóticas y laurel (Cordia alliodora), amarillón (Terminalia amozonia), surá (Terminalia oblonga), cebo (Vochysia guatemalensis), pochote (Bombacopsis quinata) y jaúl (Alnus acuminata), entre las nativas (Murillo et al. 2001, Pérez y Kanninen 2001, Petit y Montagnini 2004, Moya 2004).

Uno de los inconvenientes que presenta el mercado actual de madera de estas especies es que la madera aserrada se ha dedicado principalmente a la fabricación de tarimas (Barrantes y Ugalde 2012), aunque algunas de ellas, como melina y teca, en la actualidad son utilizadas en una gran variedad de productos (Moya 2004, Moya y Pérez 2007). En las otras especies, no se han dado los resultados esperados debido a que no se cuenta con una disponibilidad que permita establecer un mercado estable, a la falta de disponibilidad de materia prima y a la carencia de conocimientos sobre las propiedades anatómicas, físicas y mecánicas de estas maderas (Moya y Muñoz 2010, Moya et al. 2010).

Así mismo, existe un gran interés del sector forestal productivo privado de Costa Rica por conocer las propiedades de las especies con potencial para la reforestación comercial, dadas las necesidades urgentes de certificar los posibles usos y aplicaciones de este tipo de madera (Tuk 2010). El sector construcción por su with the highest strength in tension parallel to the grain. A. mangium and Cupressus lusitanica presented the highest values in shear parallel to grain. $V$. guatemalensis and Bombacopsis quinata showed lower resistance in all measured mechanical properties analyzed. The derivation of allowable stress design was possible to establish three groups of species. Group 1 is composed of A.mangium, T. grandis, T. amazonia and T. oblonga, with the highest allowable stress design. Group 2, with intermediate allowable stress design values, component species Gmelina arborea and C. Iusitanica. Group 3 species, with lower allowable values, is grouped of Alnus acuminata, $B$. quinata and $V$. guatemalensis.

Key words: Tropical wood, plantations, fast-growth, structural grade, wood resistance, Costa Rica.

parte, a pesar de ser el principal consumidor de madera aserrada en Costa Rica, está sufriendo un proceso acelerado de sustitución por materiales metálicos, plásticos y los derivados del concreto (Serrano 2006). Uno de los inconvenientes señalados al uso de la madera en la construcción, es que no se cuenta con esfuerzos admisibles de diseño para especies de plantación ni con una estandarización o clasificación de la madera usada en este ramo (Serrano y Moya 2013, Tuk 2010).

Los esfuerzos admisibles para diseño son valores de resistencia mecánica que se obtienen para los materiales y que se derivan de pruebas experimentales. En el caso de la madera, a diferencia de otros materiales, debido a su naturaleza ortotrópica y su gran variabilidad natural, los valores promedio deben reducirse por varios factores tales como defectos, variabilidad natural, contenido de humedad, tipo de esfuerzo, entre otros. Estos valores son importantes, ya que el profesional estructural requiere para sus cálculos, información confiable para proyectar las dimensiones de los elementos y las conexiones que componen la estructura tales como vigas, columnas y armaduras (Tuk 2010).

Los esfuerzos admisibles de diseño derivados para la madera de plantación de Costa Rica se obtienen mediante el siguiente procedimiento (INTECO 2010): (i) muestreo y selección de los árboles, (ii) preparación y ejecución de los ensayos mecánicos según la norma ASTM D 143-94 (ASTM 2003a) (iii) obtención del promedio y la desviación estándar de cada propiedad, (iv) cálculo del esfuerzo de referencia (corresponde con el quinto percentil), (v) ajuste del esfuerzo de referencia por grado estructural, de acuerdo con la razón de resistencia definida para cada grado, (vi) ajuste por duración de la carga y factor de seguridad y (vii) ajuste por secado.

En Costa Rica se cuenta con poca información de los esfuerzos admisibles de diseño para madera. 
Recientemente, Tuk (2010) publicó los valores de esfuerzos admisibles de diseño de 16 especies de madera proveniente del bosque natural, a pesar de que dicha información está disponible desde 1983 como parte de un proyecto de investigación del Instituto Tecnológico de Costa Rica. Así mismo, es poco conocido por los profesionales estructurales que el grupo de países Andinos (Colombia, Perú, Bolivia, Ecuador y Venezuela) cuenta con valores de esfuerzos admisibles de diseño para maderas tropicales en tres categorías de densidades (Keenan y Tejada 1987) y los mismos se pueden aplicar a las maderas de bosque natural de Costa Rica.

Ante la carencia de esfuerzos admisibles de diseño para madera proveniente de plantaciones forestales es que el presente trabajo tiene como objetivo desarrollar los valores de esfuerzos admisibles de nueve principales especies (melina, teca, acacia, laurel, amarillón, jaúl, surá, cebo y pochote) utilizadas en la reforestación comercial de Costa Rica. Los esfuerzos admisibles son calculados para flexión estática, compresión y cortante.

\section{Materiales y métodos}

\section{Plantaciones utilizadas y muestreo}

Las plantaciones muestreadas presentaron edades entre 9 y 13 años, densidades entre 338 a 575 árboles/ ha y diferentes intensidades de manejo, los cuales se detallan en el Cuadro 1. En todas las plantaciones, el espaciamiento inicial fue de $3 \times 3 \mathrm{~m}$, y una densidad inicial de 1111 árboles/ha. En todos los casos se obtuvo madera del segundo raleo de cada plantación, ya que a estas edades, la mayoría de las especies plantadas presentan el potencial de utilizar la madera para uso estructural.

En la selección de los árboles a muestrear se siguió la metodología propuesta por Moyaet al. (2002) que consiste en establecer dos parcelas temporales, distribuidas en un área de aproximadamente 1 hectárea representativa de toda la plantación. En ella fueron tomados los datos de diámetro a 1,30 $\mathrm{m}$ de altura del suelo, altura total de los árboles y altura de inicio de la copa. Posteriormente, con los datos de diámetro, se confeccionó la distribución de frecuencia acumulada de los diámetros presentes en la plantación muestreada y se dividió en tres partes correspondientes a los terciles de la distribución. Tres categorías de dominancia respecto al diámetro fueron establecidas: grueso, medio y delgado. En cada categoría se seleccionaron tres árboles al azar, dando como resultado nueve árboles por cada plantación. Se tuvo el cuidado de que los árboles fueran rectos, sin bifurcaciones y sin daños visibles. Para cada uno de los árboles seleccionados se cortó una troza entre la base y $2,5 \mathrm{~m}$ de altura. Además, a esta altura también se cortó un disco de aproximadamente $3,0 \mathrm{~cm}$ de espesor para la determinación del peso específico básico (PEB).

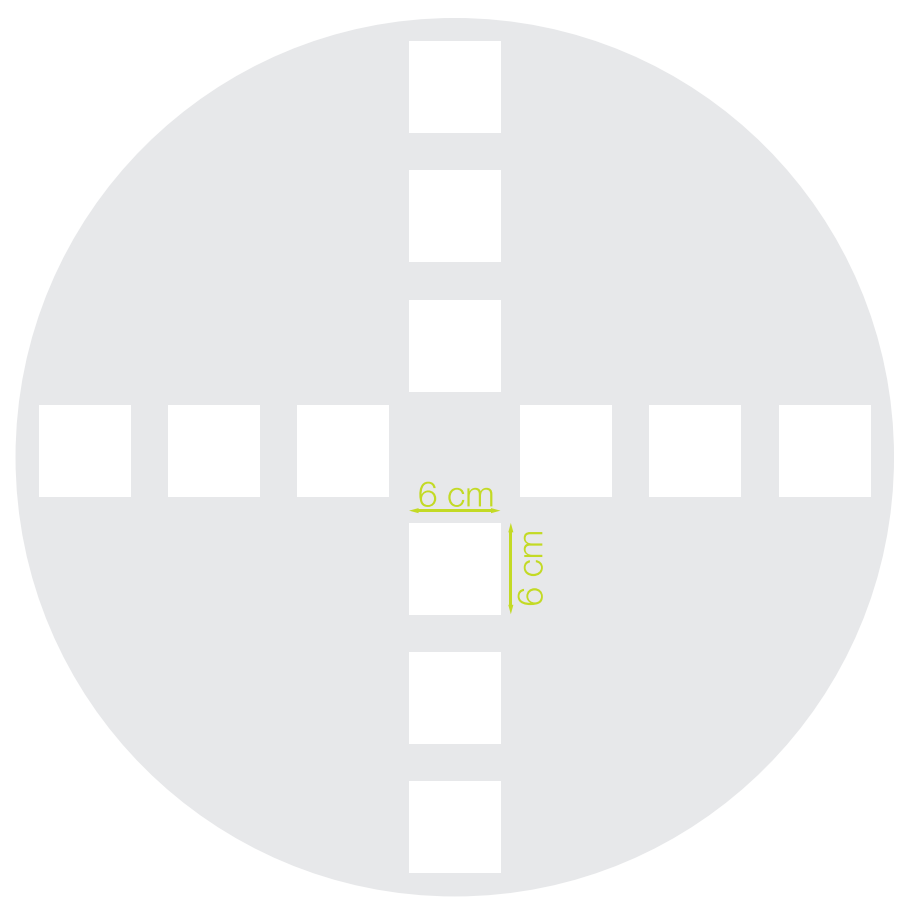

Figura 1. Esquema de corte utilizado en la obtención de las muestras para las propiedades mecánicas de la madera. Fuente: ASTM D-553694 (ASTM 2003b).

\section{Muestreo dentro del árbol}

Las propiedades mecánicas fueron evaluadas a partir de madera libre de defectos de las trozas obtenidas, a las cuales se les aplicó el patrón de corte establecido en la norma ASTM D 5536 (ASTM 2003b). Para ello, en la parte central del árbol se cortaron bloques de tipo cruciforme con dimensiones de $6 \mathrm{~cm} \times 6 \mathrm{~cm}$ de sección transversal, para luego obtener piezas de $5 \mathrm{~cm} \times 5 \mathrm{~cm}$ o bien piezas de 2,5 cm x 2,5 cm (Figura 1).

\section{Condición de humedad evaluada}

El material extraído por el muestreo (Figura 1), fue colocado en condiciones de temperatura de $22{ }^{\circ} \mathrm{C}$ y $66 \%$ de humedad relativa, lo que permitió alcanzar un contenido de humedad de equilibrio de $12 \%$, denominado como seco al aire. Los ensayos de resistencia, por lo tanto, fueron realizados en esa condición.

\section{Propiedades mecánicas evaluadas}

Las propiedades mecánicas determinadas fueron las siguientes: flexión estática $\left(F_{b}\right)$ que da como resultado el módulo de ruptura (MOR) y módulo de elasticidad $(E)$, esfuerzo en compresión paralela al grano $\left(F_{c}\right)$, esfuerzo en compresión perpendicular al grano $\left(F_{c \mathbb{Q}}\right)$, esfuerzo en cortante paralelo al grano $\left(F_{v}\right)$, esfuerzo en tensión paralela al grano $\left(F_{t}\right)$ y. Estas propiedades se determinaron según la norma ASTM D 143 (ASTM 2003a). Los especímenes que se utilizan están libres de defectos y sus dimensiones se establecen de acuerdo con la norma indicada anteriormente. De cada troza se 


\begin{tabular}{|c|c|c|c|c|c|}
\hline Especie & $\begin{array}{l}\text { Edad } \\
\text { (años) }\end{array}$ & $\begin{array}{l}\text { Densidad de } \\
\text { plantación } \\
\text { (árboles/hectárea) }\end{array}$ & Altura total $(\mathrm{m})$ & $\begin{array}{l}\text { Diámetro } \\
\text { altura de } \\
\text { pecho }(\mathrm{cm})\end{array}$ & Manejo \\
\hline Acacia mangium & 9 & 556 & 17,1 & 20,5 & $\begin{array}{l}\text { A la edad de } 4 \text { años fue aplicado un raleo del } 50 \% \\
\text { de los árboles existentes. }\end{array}$ \\
\hline Alnus acuminata & 9 & 338 & 19,0 & 26,7 & $\begin{array}{l}\text { Se aplicaron limpiezas anuales de la vegetación } \\
\text { arbustiva y a las edades de } 3 \text { y } 6 \text { años se } \\
\text { ejecutaron raleos de mantenimiento. }\end{array}$ \\
\hline Bombapcosis quinata & 15 & 240 & 24,5 & 31,3 & $\begin{array}{l}\text { Fue aplicado un raleo sanitario y } 1 \text { raleo del } 50 \% \text { a } \\
\text { la edad de } 7 \text { años }\end{array}$ \\
\hline Cupressus lusitanica & 18 & 408 & 19,24 & 28,02 & $\begin{array}{l}\text { Se aplicaron limpiezas anuales de la vegetación } \\
\text { arbustiva y a las edades de } 6 \text { y } 10 \text { años se } \\
\text { ejecutaron raleos de mantenimiento. }\end{array}$ \\
\hline Gmelina arborea & 12 & 320 & 20,4 & 26,12 & $\begin{array}{l}\text { Raleo sanitario y un raleo de } 50 \% \text { a la edad de } 4 \\
\text { y } 8 \text { años }\end{array}$ \\
\hline Tectona grandis & 13 & 475 & 21,85 & 30,2 & $\begin{array}{l}\text { Recibieron las primeras prácticas de } \\
\text { mantenimiento (raleos y podas) a la edad de } 6 \\
\text { años. }\end{array}$ \\
\hline Terminalia amazonia & 14 & 452 & 21,40 & 22,59 & $\begin{array}{l}\text { Fue aplicado un raleo sanitario y } 2 \text { raleo del } 50 \% \text { a } \\
\text { la edad de } 5 \text { y } 9 \text { años. }\end{array}$ \\
\hline Terminalia oblonga & 10 & $495-575$ & 20,70 & 18,50 & $\begin{array}{l}\text { Fue aplicado un raleo sanitario y } 1 \text { raleo del } 50 \% \text { a } \\
\text { la edad de } 8 \text { años. }\end{array}$ \\
\hline Vochysia guatemalensis & 8 & 515 & 22,7 & 18,5 & $\begin{array}{l}\text { A la edad de } 4 \text { años fue aplicado un raleo del } 50 \% \\
\text { de los árboles existentes. }\end{array}$ \\
\hline
\end{tabular}

obtienen un total de 4 muestras por tipo de ensayo para un total de 36 especímenes ensayados.

Para la determinación del peso específico básico (PEB) se utiliza el disco obtenido a una altura de 2,5 m. Del disco se cortan dos secciones para eliminar la médula y la medición se realiza sobre ambas. El peso específico básico fue determinado según la norma ASTM D 2395 (ASTM 2003c).

\section{Derivación de los esfuerzos admisibles de diseño}

Los esfuerzos admisibles de diseño fueron calculados para cada especie y para tres grados estructurales, denominados como grado 1 , grado 2 y grado 3 . El grado 1 representan la madera estructural de mayor resistencia para la especie, el grado 3 la madera estructural de menor resistencia y el grado 2, la madera estructural de resistencia intermedia entre el grado 1 y 3.

La derivación de los esfuerzos admisibles se basó en lo indicado en la norma ASTM D 245 (ASTM 2003d): (i) se calculan el promedio y la desviación estándar de los ensayos de resistencia realizados, (ii) se obtiene el esfuerzo de referencia que corresponde a la exclusión del quinto percentil, (iii) se ajusta el esfuerzo de referencia por grado estructural de acuerdo con la razón de resistencia definida para cada grado (Cuadro 2), (iv) se aplica un ajuste por duración de la carga y factor de seguridad (Cuadro 2), (v) se aplica un factor de ajuste por secado.
Este factor se debe a que los ensayos se realizaron con una condición del $12 \%$ de contenido de humedad, y estos deben reportarse para un contenido de humedad de $19 \%$. (vi) Finalmente, se debe aplicar un factor de tamaño $C_{F}$ al esfuerzo admisible de flexión $\left(F_{b}\right)$ que depende de la altura $d$ de la sección que se vaya a emplear:

$$
C_{F}=\left(\frac{50}{d}\right)^{1 / 9}
$$

En la ecuación anterior, $d$ debe estar en $\mathrm{mm}$. El número 50 aparece debido a que los ensayos de flexión se realizan a especímenes de $50 \mathrm{~mm}$ de altura.

\section{Análisis estadísticos}

La normalidad y la presencia de valores atípicos fueron verificadas para cada propiedad mecánica en la madera libre de defectos. Posteriormente se aplicó un análisis de la varianza (ANOVA) en propiedades mecánicas, donde la variable independiente correspondió a la especie y como variable de respuesta las propiedades analizadas. Finalmente para encontrar diferencias estadísticas entre las medias se aplicó la prueba de Tukey, al 1,0\% de significación. 


\begin{tabular}{|c|c|c|c|c|c|}
\hline \multirow{2}{*}{ Propiedad } & \multicolumn{3}{|c|}{ Grados estructurales } & \multirow{2}{*}{$\begin{array}{l}\text { Factor por duración } \\
\text { de carga y factor de } \\
\text { seguridad }\end{array}$} & \multirow{2}{*}{ Ajuste por secado } \\
\hline & Grado 1 & Grado 2 & Grado 3 & & \\
\hline Flexión (Fb) & 70 & 55 & 40 & $1 / 2,1$ & 0,86 \\
\hline Módulo de elasticidad (E) & 100 & 90 & 80 & $1 / 0,94$ & 0,97 \\
\hline Compresión paralela al grano (Fc) & 70 & 55 & 40 & $1 / 1,9$ & 0,67 \\
\hline Compresión Perpendicular al grano ( $\left.\mathrm{Fc}^{\perp} \perp\right)$ & 100 & 100 & 100 & $1 / 1,67$ & 0,7 \\
\hline Cortante paralelo al grano (Fv) & 50 & 50 & 50 & $1 / 2,1$ & 0,97 \\
\hline Tensión paralela al grano (Ft) & 40 & 30 & 20 & $1 / 2,1$ & 0,84 \\
\hline
\end{tabular}

\section{Resultados y discusión}

\section{Valores de resistencia en madera libre de defectos}

Los promedios de las propiedades mecánicas ejecutadas para derivar los esfuerzos admisibles de diseño son mostrados en el Cuadro 3. Las propiedades de $T$. grandis, $A$. mangium, las dos especies de Terminalia y $C$. lusitanica presentaron valores de resistencia más altos en algunas propiedades en relación a las otras especies. En tanto que, $V$. guatemalensis y $B$. quinata obtuvieron los valores más bajos de las propiedades medidas.

Un aspecto importante a destacar en los valores de resistencia encontrados, es la relación de entre estos y los valores de PEB. Por ejemplo, V. guatemalensis y $B$. quinata, con los valores de PEB más bajos, de 0,36 y 0,35 respectivamente, son las especies de más baja resistencia en todas las propiedades. En tanto que las especies con altos valores de PEB, presentaron en general alta resistencia mecánica. Pero se evidencia un patrón confuso entre las especies de valores altos de PEB, $A$. mangium, C. lusitanica, $T$. oblonga y $T$. amazonia. Por ejemplo, $T$. amazonia presenta un PEB similar que $A$. mangium (0,58 y 0,57 respectivamente), T. amazonia promedió $17,9 \mathrm{MPa}$ en la resistencia a la compresión paralela a la fibra, mientras que en la especie A. mangium en esa misma propiedad presentó un valor significativamente mayor, 34,0 MPa (Cuadro 3).

Estudios previos sobre los valores de resistencia de algunas de las especies estudiadas muestran valores de resistencia mayor a las encontradas en el presente estudio (Llach 1971, González et al. 1973, Keenan y Tejada 1987, Shupe et al. 2005). Esta diferencia en la resistencia mecánica es atribuida a las diferentes condiciones de crecimiento, específicamente si los árboles se desarrollan o no en plantaciones forestales. Los árboles creciendo en condiciones de plantaciones producen madera con menor PEB y por tanto propiedades de resistencia inferior (Zobel y Sprague 1998). Por ejemplo, árboles de T. amazonia creciendo en bosques naturales de Panamá (Llach 1971), Nicaragua (González et al. 1973), Honduras (Shupe et al. 2005), Bolivia, Venezuela y Colombia (Keenan y Tejada 1987) reportan propiedades mecánicas superiores a las encontradas en los árboles estudiados que provienen de plantaciones forestales. En el caso de V. guatemalensis, los árboles creciendo en bosques naturales en La Ceiba (Honduras), Panamá (Llach 1971), Nicaragua (González et al. 1973), Honduras (Shupe et al. 2005), y Costa Rica (Tuk 1980) demostraron resistencia más altas en las propiedades mecánicas que los reportadas por Sotela y Carpio (1991) y el presente estudio.

Aunque fue encontrado en este estudio que los árboles de plantaciones presentaron una resistencia más baja que aquellos árboles creciendo en los bosques naturales, algunas propiedades como el PEB, incrementó con la edad de los árboles (Zobel y Sprague 1998). Estos comportamientos son atribuidos a que muchos cambios moleculares y fisiológicos ocurren en el cambio vascular durante el envejecimiento (Plomion et al. 2001). Por ejemplo, durante la etapa adulta, la pared celular de las fibras es más gruesa que la pared de las fibras producidas en la etapa juvenil (Horacek et al. 1999), por lo que es de esperar que los valores de resistencia de las especies estudiadas aumenten en árboles cosechados en el tercer raleo o cosecha final de las plantaciones y por tanto los valores de esfuerzos admisibles de diseño pueden variar en esas edades.

\section{Esfuerzos admisibles de diseño}

La derivación de los esfuerzos admisibles de diseño de las diferentes propiedades de las especies de plantación estudiadas se presentan en el Cuadro 4. Dichos valores son derivados según la aplicación de varios ajustes tal y como se describió anteriormente (ver anexo 1). En el Cuadro 3 se presentan los valores para los tres grados estructurales propuestos (grado 1, 2 y 3), en donde la resistencia disminuye con el aumento en el número de grado estructural, o sea, el grado estructural 1 presenta mayor resistencia que el grado estructural 3.

Al comparar los resultados de valores de esfuerzos admisibles de diseño con los valores admisibles de otras especies de madera de Costa Rica, pero proveniente de árboles creciendo en el bosque natural (Tuk 2010), se tiene que en el caso de la madera de Alnus acuminata y Vochysia guatemalensis, proveniente de árboles 


\begin{tabular}{|c|c|c|c|c|c|c|c|c|c|c|}
\hline Propiedad mecánica & & $\begin{array}{c}\text { Acacia } \\
\text { mangium }\end{array}$ & $\begin{array}{c}\text { Alnus } \\
\text { acuminata }\end{array}$ & $\begin{array}{c}\text { Bombapcosis } \\
\text { quinata }\end{array}$ & $\begin{array}{l}\text { Cupressus } \\
\text { lusitanica }\end{array}$ & $\begin{array}{c}\text { Gmelina } \\
\text { arborea }\end{array}$ & $\begin{array}{l}\text { Tectona } \\
\text { grandis }\end{array}$ & $\begin{array}{l}\text { Terminalia } \\
\text { amazonia }\end{array}$ & $\begin{array}{c}\text { Terminalia } \\
\text { oblonga }\end{array}$ & $\begin{array}{c}\text { Vochysia } \\
\text { guatemalensis }\end{array}$ \\
\hline Peso específico básico & & 0,57 & 0,39 & 0,35 & 0,54 & 0,45 & 0,58 & 0,58 & 0,61 & 0,36 \\
\hline \multirow{2}{*}{ Flexión estática (MPa) } & MOR & $\begin{array}{l}78,4^{\mathrm{ab}} \\
(1,22)\end{array}$ & $\begin{array}{l}51,9^{c} \\
(1,93)\end{array}$ & $\begin{array}{l}35,8^{d} \\
(2,01)\end{array}$ & $\begin{array}{l}57,6^{c} \\
(1,85)\end{array}$ & $\begin{array}{l}95,1^{\mathrm{a}} \\
(8,17)\end{array}$ & $\begin{array}{l}50,3^{c} \\
(0,87)\end{array}$ & $\begin{array}{l}74,6^{b} \\
(1,58)\end{array}$ & $\begin{array}{l}82,3^{a b} \\
(0,88)\end{array}$ & $\begin{array}{l}40,4^{d} \\
(1,03)\end{array}$ \\
\hline & MOE & $\begin{array}{l}12310^{\mathrm{a}} \\
(1010)\end{array}$ & $\begin{array}{l}7700^{\circ} \\
(2310)\end{array}$ & $\begin{array}{l}5600^{e} \\
(1990)\end{array}$ & $\begin{array}{l}7057^{\circ} \\
(2720)\end{array}$ & $\begin{array}{l}13100^{a} \\
(1120)\end{array}$ & $\begin{array}{l}8300^{\circ} \\
(1490)\end{array}$ & $\begin{array}{c}11910^{a} \\
(904)\end{array}$ & $\begin{array}{l}8068^{b} \\
(1372)\end{array}$ & $\begin{array}{l}6410^{d} \\
(1070)\end{array}$ \\
\hline \multirow{2}{*}{ Compresión (MPa) } & // & $\begin{array}{l}34^{\mathrm{bc}} \\
(3,37)\end{array}$ & $\begin{array}{c}29^{c} \\
(1,44)\end{array}$ & $\begin{array}{c}8,7^{f} \\
(2,29)\end{array}$ & $\begin{array}{l}14,3^{e} \\
(1,56)\end{array}$ & $\begin{array}{l}42,5^{\mathrm{a}} \\
(4,51)\end{array}$ & $\begin{array}{l}31,8^{b} \\
(5,86)\end{array}$ & $\begin{array}{l}17,9^{d} \\
(1,20)\end{array}$ & $\begin{array}{l}37,5^{b} \\
(0,84)\end{array}$ & $\begin{array}{l}21,3^{d} \\
(1,31)\end{array}$ \\
\hline & $\perp$ & $\begin{array}{c}24^{c} \\
(2,52)\end{array}$ & $\begin{array}{l}15,6^{d} \\
(1,80)\end{array}$ & $\begin{array}{l}10,7^{e} \\
(2,40)\end{array}$ & $\begin{array}{l}24,1^{c} \\
(1,65)\end{array}$ & $\begin{array}{l}39,6^{a} \\
(1,05)\end{array}$ & - & $\begin{array}{l}22,3^{c} \\
(2,23)\end{array}$ & $\begin{array}{l}35,3^{b} \\
(1,16)\end{array}$ & $\begin{array}{l}14,5^{d} \\
(1,58)\end{array}$ \\
\hline $\begin{array}{l}\text { Cortante paralela al grano } \\
\text { (MPa) }\end{array}$ & & $\begin{array}{l}9,6^{a} \\
(1,5)\end{array}$ & $\begin{array}{c}7,9^{b} \\
(2,13)\end{array}$ & $\begin{array}{c}4,7^{d} \\
(1,71)\end{array}$ & $\begin{array}{c}9,4^{\mathrm{a}} \\
(1,54)\end{array}$ & $\begin{array}{c}6,3^{c} \\
(1,00)\end{array}$ & $\begin{array}{c}6,2^{c} \\
(1,05)\end{array}$ & $\begin{array}{c}7,5^{\mathrm{b}} \\
(2,57)\end{array}$ & $\begin{array}{c}7,6^{b} \\
(0,78)\end{array}$ & $\begin{array}{c}6,3^{c} \\
(1,64)\end{array}$ \\
\hline $\begin{array}{l}\text { Tensión paralela al grano } \\
\text { (MPa) }\end{array}$ & & $\begin{array}{l}89,7^{\mathrm{a}} \\
(2,08)\end{array}$ & $\begin{array}{l}67,7^{\mathrm{bc}} \\
(2,44)\end{array}$ & $\begin{array}{l}43,0^{d} \\
(3,29)\end{array}$ & $\begin{array}{l}52,2^{\text {cd }} \\
(3,87)\end{array}$ & $\begin{array}{l}47,0^{d} \\
(8,00)\end{array}$ & $\begin{array}{l}91,2^{\mathrm{a}} \\
(20,1)\end{array}$ & $\begin{array}{l}56,7^{\mathrm{bcd}} \\
(2,72)\end{array}$ & $\begin{array}{l}65,1^{1 \mathrm{c}} \\
(2,85)\end{array}$ & $\begin{array}{l}39,4^{d} \\
(2,77)\end{array}$ \\
\hline
\end{tabular}

Leyenda: // = paralelo al grano, $\perp$ = perpendicular al grano.

Nota: Los valores entre paréntesis representa la desviación estándar y letras diferentes entre especies en una misma propiedad son estadísticamente diferentes en un nivel de confianza de $99 \%$.

creciendo en bosques naturales, se reportan valores de esfuerzos admisibles de diseño ligeramente mayor o similar que los que presenta la madera de los árboles creciendo en condiciones de plantaciones forestales, que es el caso de este estudio. Por ejemplo, en el valor de módulo de elasticidad $(E)$ encontrado en la madera de plantaciones de Alnus acuminata de este estudio es de $7500 \mathrm{MPa}$ en el grado estructural 1, mientras que la madera de esa especie pero proveniente de bosque natural, el valor del esfuerzo admisible de diseño reportado es $11500 \mathrm{MPa}$. En tanto que la madera de Vochysia guatemalensis de bosque natural presenta un esfuerzo en flexión $\left(F_{b}\right)$ de $9,5 \mathrm{MPa}$ en el grado estructural 1 , pero en plantaciones, para este mismo grado estructural 1, presenta un valor de 11,4 MPa.

En el estudio llevado a cabo por Tuk (2010) se establecieron las siguientes categorías: especies duras, semiduras, suaves y muy suaves. Al considerar esta misma clasificación se tiene que ninguna de las especies de plantación estudiada presenta valores comparables al grupo de las especies duras. En tanto que $T$. grandis, A. mangium, $T$. amazonia y $T$. oblonga presenta valores de esfuerzos admisibles de diseño comparables con el grupo de especies de madera semiduras. Las especies como G. arborea y C. lusitanica, presentaron valores admisibles similares a las especies suaves y finalmente $V$. guatemalensis, $A$. acuminata y $B$. quinata presentaron valores de resistencia similares a las especies catalogadas como muy suaves.

Basados en los resultados anteriores, se puede establecer una separación en tres grupos de diferentes especies para las maderas de plantación de Costa Rica. En un primer grupo se ubican las especies con los valores más altos de esfuerzos admisibles de diseño en las diferentes propiedades y lo componen $A$. mangium, $T$. grandis, T. amazonia y $T$. oblonga. El segundo grupo, con valores intermedios de valores admisibles, lo componen las especies G. arborea y C. lusitanica. Finalmente, el grupo de especies con los valores admisibles más bajos es compuesto por $A$. acuminata, $B$. quinata y $V$. guatemalensis (Cuadro 4).

Al comparar esta posible separación de grupos de especies con otras maderas de diferentes latitudes, por ejemplo, la clasificación de la Junta del Acuerdo de Cartagena (JUNAC), que trabajó varios grupos de especies del sector Andino de América del Sur (Keenan y Tejada 1987), se tiene que las especies de plantación de Costa Rica con mayores esfuerzos admisibles de diseño (A. mangium, T. grandis, T. amazonia y $T$. oblonga), presentaron valores de esfuerzos admisibles similares al grupo estructural B de la JUNAC. Situación que es de esperar porque la JUNAC ubica en ese grupo a especies con PEB de 0,56 a 0,70 y las especies de plantación de $A$. mangium, $T$. grandis, $T$. amazonia y $T$. oblonga presentan un PEB dentro de ese rango (Cuadro 4). Asimismo, el grupo intermedio en este estudio, compuesto por G. arborea y C. Iusitanica, es comparable con el grupo estructural $\mathrm{C}$, que incluye todas las especies con valores de PEB entre 0,40 a 0,55 , rango que contempla los valores encontrados en las anteriores dos especies (Cuadro 4). Finalmente, la JUNAC no estima esfuerzos admisibles de diseño para especies con PEB menor de 0,40. Sin embargo, en el estudio de las maderas de plantaciones de Costa Rica, existen tres especies ( $A$. acuminata, $B$. quinata y $V$. guatemalensis) en las que los esfuerzos admisibles llegan a alcanzar los valores de la menor categoría del grupo estructural 
Cuadro 4. Esfuerzos admisibles de diseño para nueve especies de plantación de Costa Rica secada a 12\% de contenido de humedad.

\begin{tabular}{|c|c|c|c|c|c|c|c|c|}
\hline \multirow{2}{*}{ Especie } & \multicolumn{7}{|c|}{ Esfuerzos admisibles para cada grado estructural(MPa) } & \multirow{2}{*}{$\begin{array}{c}\text { Peso } \\
\text { específico } \\
\text { Básico }\end{array}$} \\
\hline & $\mathrm{Fb}$ & E & Emin & Fc & $\mathrm{Fc}_{\perp}$ & Fv & $\mathrm{Ft}$ & \\
\hline \multicolumn{8}{|l|}{ Acacia (Acacia mangium) } & \multirow{4}{*}{0,57} \\
\hline Grado 1 & 17,2 & 12000 & 10000 & 6,7 & 1,34 & 11,34 & 11,20 & \\
\hline Grado 2 & 13,4 & 9000 & 10500 & 5,2 & 1,34 & 8,4 & 11,94 & \\
\hline Grado 3 & 9,8 & 8000 & 9500 & 3,8 & 1,34 & 5,6 & 11,94 & \\
\hline \multicolumn{8}{|l|}{ Jaúl (Alnusacuminata) } & \multirow{4}{*}{0,39} \\
\hline Grado 1 & 11,4 & 7500 & 3500 & 5,7 & 7,52 & 0,78 & 8,4 & \\
\hline Grado 2 & 9,0 & 6500 & 3000 & 4,5 & 7,52 & 0,78 & 6,3 & \\
\hline Grado 3 & 6,5 & 6000 & 2500 & 3,2 & 7,52 & 0,78 & 4,2 & \\
\hline \multicolumn{8}{|c|}{ Pochote (Bombapcosis quinata) } & \multirow{4}{*}{0,35} \\
\hline Grado 1 & 7,8 & 2000 & 5500 & 1,0 & 0,34 & 5,4 & 3,64 & \\
\hline Grado 2 & 6,2 & 2000 & 5000 & 0,8 & 0,34 & 4,0 & 3,64 & \\
\hline Grado 3 & 4,5 & 1500 & 4500 & 0,6 & 0,34 & 2,7 & 3,64 & \\
\hline \multicolumn{8}{|l|}{ Ciprés (Cupressus lusitanica) } & \multirow{4}{*}{0,54} \\
\hline Grado 1 & 12,6 & 2500 & 7500 & 2,7 & 1,30 & 6,5 & 12,18 & \\
\hline Grado 2 & 9,8 & 2500 & 6500 & 2,1 & 1,30 & 4,9 & 12,18 & \\
\hline Grado 3 & 7,2 & 2000 & 6000 & 1,6 & 1,30 & 3,3 & 12,18 & \\
\hline \multicolumn{8}{|l|}{ Melina (Gmelina arborea) } & \multirow{4}{*}{0,45} \\
\hline Grado 1 & 11,0 & 8000 & 5000 & 4,8 & - & 0,88 & 4,7 & \\
\hline Grado 2 & 8,6 & 7000 & 4500 & 3,7 & - & 0,88 & 3,5 & \\
\hline Grado 3 & 6,3 & 6500 & 4000 & 2,7 & - & 0,88 & 2,3 & \\
\hline \multicolumn{8}{|l|}{ Teca (Tectona grandis) } & \multirow{4}{*}{0,58} \\
\hline Grado 1 & 20,8 & 10500 & 12500 & 8,0 & 0,84 & 7,8 & 4,72 & \\
\hline Grado 2 & 16,4 & 9500 & 11500 & 6,2 & 0,84 & 5,8 & 4,72 & \\
\hline Grado 3 & 11,8 & 8500 & 10000 & 4,5 & 0,84 & 3,9 & 4,72 & \\
\hline \multicolumn{8}{|c|}{ Roble coral (Terminalia amazonia) } & \multirow{4}{*}{0,54} \\
\hline Grado 1 & 16,4 & 11500 & 9500 & 3,5 & 0,16 & 7,0 & - & \\
\hline Grado 2 & 12,8 & 10500 & 8500 & 2,8 & 0,16 & 5,3 & - & \\
\hline Grado 3 & 9,4 & 9000 & 7500 & 2,0 & 0,16 & 3,5 & - & \\
\hline \multicolumn{8}{|l|}{ Amarillon (Terminalia oblonga) } & \multirow{4}{*}{0,61} \\
\hline Grado 1 & 18,0 & 8000 & 5500 & 7,4 & 1,28 & 8,2 & 17,84 & \\
\hline Grado 2 & 14,2 & 7000 & 4500 & 5,8 & 1,28 & 6,1 & 17,84 & \\
\hline Grado 3 & 10,2 & 6000 & 4000 & 4,2 & 1,28 & 4,1 & 17,84 & \\
\hline \multicolumn{8}{|c|}{ Chancho blanco Nochysia guatemalensis) } & \multirow{4}{*}{0,36} \\
\hline Grado 1 & 8,8 & 6000 & 4000 & 4,2 & 0,64 & 4,9 & 7,12 & \\
\hline Grado 2 & 7,0 & 5500 & 4000 & 3,3 & 0,64 & 3,7 & 7,12 & \\
\hline Grado 3 & 5,0 & 5000 & 3500 & 2,4 & 0,64 & 2,5 & - & \\
\hline
\end{tabular}

Donde: Fb: Esfuerzo en flexión, E: Módulo de elasticidad, Emin: Módulo de elasticidad mínimo, Fc: Esfuerzo en compresión paralela, Fc ${ }_{\perp}$ : Esfuerzo en compresión perpendicular al grano, Fv: Esfuerzo en cortante, Ft: Esfuerzo en tensión paralela al grano.

Nota:

1. Ios valores de este cuadro se derivan para ser utilizados con la metodología de diseño presentada en la especificación "National Design Specificationfor Wood Construction ASD/LRFD"

2. El peso específico básico corresponde a la relación del peso seco al horno y el volumen en estado verde. 
C de la JUNAC. Además, en ninguna de las especies estudiadas se encontró valores de esfuerzo admisibles de diseño próximos al grupo estructural $A$ de la JUNAC (con especies que presenten valores de PEB de 0,71 a $0,90)$, debido a que ninguna de las especies presenta valores de PEB superior a 0,61 (Cuadro 4).

\section{Conclusiones}

Laderivación de los esfuerzos de diseño para las principales especies de plantación utilizadas en la reforestación comercial de Costa Rica, permitió establecer tres grupos de especies. El grupo 1 lo componen las especies $A$. mangium, $T$. grandis, $T$. amazonia y $T$. oblonga con los valores más altos de esfuerzos admisibles de diseño en las diferentes propiedades. El grupo 2, con valores intermedios de valores admisibles, lo componen $G$. arborea y $C$. lusitanica. Finalmente, el grupo 3 presentaron los valores admisibles más bajos y estába compuesto por $A$. acuminata, $B$. quinata $V$. guatemalensis.

Esta información permite disponer de valores de diseño confiable para los diseños estructurales correspondientes, pero también obliga a que los productores entrenen a su personal para que realicen una clasificación visual acertada.

\section{Agradecimientos}

A la Vicerrectoría de Investigación y Extensión del Instituto Tecnológico de Costa Rica por el apoyo financiero al Proyecto de Investigación.

\section{Referencias}

ASTM (American Society for Testing and Materials, US). 2003a. D 143-94. Standard test methods for small clear specimens of timber. In Annual Book of ASTM Standards. Philadelphia, US, ASTM. Vol. 04.10. 31 p.

ASTM (American Society for Testing and Materials, US). 2003b. D5536-94. Standard practice for sampling forest trees for determination of clear wood Properties. In Annual Book of ASTM standards. Philadelphia, US, ASTM. Vol. 11.06. 9 p. (ASTM D 5536-94 Reproved 2004).

ASTM (American Society for Testing and Materials, US). 2003c. D 2395-02 Standard test methods for specific gravity of wood and wood-base materials. In Annual Book of ASTM Standards. Philadelphia, US, ASTM. Vol. 04.10. 7 p.

ASTM (American Society for Testing and Materials, US). 2003d. D 2555-02 Standard test methods for establishing clear wood strength values. In Annual Book of ASTM Standards. Philadelphia, US, ASTM. Vol. 04.10. 16 p.

Barrantes, A., Ugalde S. 2012. Usos y aportes de la madera en Costa Rica. Estadísticas 2011. San José, CR, Oficina Nacional Forestal. p.9
Castro, R., Tattembach F., Arias G. 1998. Costa Rica: hacia la sostenibilidad de sus recursos naturales. San José, CR, Fondo Nacional de Financiamiento Forestal. pp. 6-7

González, G., Wiessel C., Chavarria G. 1973. Propiedades y usos de quince especies maderables del nordeste de Nicaragua. Managua, NI, Programa de las Naciones Unidas FO: SF/NIC 9. p. 166 (Informe Técnico n9).

INTECO (Instituto de Normas Técnicas de Costa Rica). 2010. Madera estructural - Clasificación en grados estructurales para la madera aserrada mediante una evaluación visual. San José, CR, INTECO. p. 7-11

Horacek, P., Slezingerova J., Gandelova L. 1999. Effect of environment on the xylogenesis of Norway spruce (Picea abies [L.]Karst.). In Wimmer, R.,Vetter R.E., (Eds). Tree-ring analysis: biological, methodological and environmental aspects. London, UK, CAB International. pp. 33-53.

Keenan, F.J., Tejada M. 1987. Maderas tropicales como material de construcción en los países del grupo andino de América del Sur. Ottawa, CA, Centro Internacional de Inv. para el Desarrollo. p. 69-89

Llach, L. 1971. Propiedades físicas y mecánicas de ciento trece especies maderables de Panamá. Parte 3. San José, Universidad de Costa Rica, Lab. de Productos Forestales. pp. 455-540.

Moya, R. 2004. Wood of Gmelina arborea in Costa Rica. New Forest 28:299-307.

Moya, R., Muñoz F. 2010. Physical and mechanical properties of eight species from fast-growth plantation in Costa Rica. Journal of Tropical Forest Science 22(3):317-328.

Moya, R., Muñoz F., Salas J., Berrocal A., Leandro L., Esquivel E. 2010. Tecnología de madera de plantaciones forestales: Fichas Técnicas. (en línea). Cartago, CR, Editorial Corporación Garro y Moya. Revista Forestal Mesoamericana Kurú 7(18-19):1-207. Consultado 5 jul. 2013. Disponible en http://www.tec-digital.itcr.ac.crl servicios/ojs/index.php/kuru/article/view/383/316

Moya, R.,Pérez D. 2007. Processing and marketing of teakwood products from fast-grown teak plantations in Costa Rica. In Regional Workshop Products and Marketing of Teak Wood Products of Planted Forest. (2007, Peechi, IN). Abstracts. Eds. Nair, K., Bhat K. V., Anitha V. Peechi, IN, KFRI. p. 24.

Moya, R., Valenzuela L., Salazar F. 2002. Efecto de la fertilización de la pradera sobre la densidad básica de Pinus radiata. D. Don. Revista Investigación Agraria: Sistemas y Recursos Forestales 11(2):182-192.

Murillo, O., Obando G., Badilla J., Sánchez S. 2001. Perspectivas en las zonas altas de Costa Rica. Biocenosis 15(1-2):6671.

Pérez, D., Kanninen M. 2001. Stand growth scenarios for Bombacopsis quinata plantations in Costa Rica. Forest Ecology \& Management 177:427-439.

Plomion, C., Leprovost G., Stokes A. 2001. Wood formation in trees. Plant Physiology 127:1513-1523.

Petit, B., Montagnini F. 2004. Growth equations and rotation ages of ten native tree species in mixed and pure plantations in the humid neotropics. Forest Ecology \& Management 199:243-257. 
Sader, S.A., Joyce A. 1988. Deforestation rates and trends in Costa Rica, 1940 to 1983. Biotropica 20:11-19.

Serrano, J. R. 2006. Consumo de madera para construcción en Costa Rica y requerimientos para satisfacer a los usuarios. (en línea). Kurú: Revista Forestal 3(8):1-6. Consultado 5 enero 2013. Disponible en http://www. tec-digital.itcr.ac.cr/servicios/ojs/index.php/kuru/article/ view/515

Serrano, J. R., Moya R. 2013. Procesamiento, uso y mercado de la madera en Costa Rica: aspectos históricos y análisis crítico. Madrid, ES, Editorial Académica Española. p. 15

Sotela, J., Carpio M. 1991. Vochysia guatemalensis en Centroamérica. Informe INII-63-1991. San José, CR, Universidad de Costa Rica, Lab. de Productos Forestales, Inst. de Investigaciones en Ingeniería. p. 11-12.

Shupe, T.F., Aguilar R.T., Vlosky R.T., Belisle M., Chávez A. 2005. Wood properties of selected lesser-used honduran wood species. Journal of Tropical Forest Science 17:438-446.

Tuk, J. 1980. Informe general del proyecto: Clasificación y normalización de maderas para uso estructural. Cartago, CR, Instituto Tecnológico de Costa Rica, Centro de Investigaciones de Ing. en Maderas. p. 11.

Tuk, J. 2010. Madera, diseño y construcción. $2^{a}$ ed. San José, $\mathrm{CR}$, Colegio Federado de Ingenieros y de Arquitectos de Costa Rica. p. 87-100

Zobel, B., Sprague J. 1998. Juvenile wood in trees. New- York, US, Springer-Verlag. p. 147-148. 


\begin{tabular}{|c|c|c|c|c|c|c|c|c|c|c|c|}
\hline Propiedad & $\begin{array}{l}\text { Promedio } \\
\text { (MPa) }\end{array}$ & $\begin{array}{l}\text { Desviación } \\
\text { Estándar } \\
\text { (MPa) }\end{array}$ & $\begin{array}{l}\text { Quinto } \\
\text { Percentil } \\
\text { (MPa) }\end{array}$ & $\begin{array}{c}\text { Razón de } \\
\text { Resistencia } \\
\text { G1 }\end{array}$ & $\begin{array}{l}\text { Razón de } \\
\text { Resistencia } \\
\text { G2 }\end{array}$ & $\begin{array}{c}\text { Razón de } \\
\text { Resistencia } \\
\text { G3 }\end{array}$ & $\begin{array}{c}\text { Factor } \\
\text { de ajuste }\end{array}$ & $\begin{array}{l}\text { Ajuste } \\
\text { por } \\
\text { secado }\end{array}$ & Grado 1 & Grado 2 & Grado 3 \\
\hline \multicolumn{12}{|c|}{ Acacia (Acacia mangium) } \\
\hline$F_{b}$ & 78,4 & 1,22 & 66,20 & 0,7 & 0,55 & 0,4 & 0,435 & 0,851 & 17,1 & 13,5 & 9,8 \\
\hline$E$ & 12310 & 1010 & 12310 & 1,0 & 0,9 & 0,8 & 1,064 & 0,905 & 11851 & 10666 & 9481 \\
\hline$E_{\min }$ & 12310 & 1010 & 10395 & 1,0 & 0,9 & 0,8 & 1,064 & 0,905 & 10008 & 9007 & 8006 \\
\hline $\mathrm{F}_{\mathrm{c}}$ & 34,0 & 3,37 & 28,71 & 0,7 & 0,55 & 0,4 & 0,476 & 0,696 & 6,7 & 5,2 & 3,8 \\
\hline $\mathrm{FC}_{\perp}$ & 24,0 & 2,52 & 19,95 & 1,0 & 1,0 & 1,0 & 0,599 & 1,000 & 12,0 & 12,0 & 12,0 \\
\hline $\mathrm{F}_{\mathrm{v}}$ & 9,6 & 1,52 & 6,73 & 0,5 & 0,5 & 0,5 & 0,435 & 0,920 & 1,3 & 1,3 & 1,3 \\
\hline$F_{t}$ & 89,7 & 2,08 & 75,74 & 0,4 & 0,3 & 0,2 & 0,435 & 0,851 & 11,2 & 8,4 & 5,6 \\
\hline \multicolumn{12}{|c|}{ Jaúl (Alnus acuminata) } \\
\hline $\mathrm{F}_{\mathrm{b}}$ & 51,9 & 1,93 & 43,83 & 0,7 & 0,55 & 0,4 & 0,435 & 0,851 & 11,4 & 8,9 & 6,5 \\
\hline$E$ & 7700 & 2310 & 7700 & 1,0 & 0,9 & 0,8 & 1,064 & 0,905 & 7413 & 6672 & 5930 \\
\hline $\mathrm{E}_{\min }$ & 7700 & 2310 & 3435 & 1,0 & 0,9 & 0,8 & 1,064 & 0,905 & 3307 & 2976 & 2645 \\
\hline $\mathrm{F}_{\mathrm{c}}$ & 29 & 1,44 & 24,49 & 0,7 & 0,55 & 0,4 & 0,476 & 0,696 & 5,7 & 4,5 & 3,2 \\
\hline $\mathrm{FC}_{\perp}$ & 7,9 & 2,13 & 3,88 & 0,5 & 0,5 & 0,5 & 0,435 & 0,920 & 0,8 & 0,8 & 0,8 \\
\hline$F_{v}$ & 67,7 & 2,44 & 57,17 & 0,4 & 0,3 & 0,2 & 0,435 & 0,851 & 8,5 & 6,3 & 4,2 \\
\hline$F_{t}$ & 15,6 & 1,8 & 12,54 & 1,0 & 1,0 & 1,0 & 0,599 & 1,000 & 8,0 & 8,0 & 8,0 \\
\hline \multicolumn{12}{|c|}{ Pochote (Bombapcosis quinata) } \\
\hline $\mathrm{F}_{\mathrm{b}}$ & 35,8 & 2,01 & 30,23 & 0,7 & 0,55 & 0,4 & 0,435 & 0,851 & 7,8 & 6,2 & 4,5 \\
\hline$E$ & 5600 & 1990 & 5600 & 1,0 & 0,9 & 0,8 & 1,064 & 0,905 & 5391 & 4852 & 4313 \\
\hline$E_{\min }$ & 5600 & 1990 & 2093 & 1,0 & 0,9 & 0,8 & 1,064 & 0,905 & 2015 & 1814 & 1612 \\
\hline$F_{c}$ & 8,7 & 2,29 & 4,36 & 0,7 & 0,55 & 0,4 & 0,476 & 0,696 & 1,0 & 0,8 & 0,6 \\
\hline $\mathrm{FC}_{\perp}$ & 10,7 & 2,4 & 6,08 & 1,0 & 1,0 & 1,0 & 0,599 & 1,000 & 3,6 & 3,6 & 3,6 \\
\hline $\mathrm{F}_{\mathrm{v}}$ & 4,7 & 1,71 & 1,71 & 0,5 & 0,5 & 0,5 & 0,435 & 0,920 & 0,3 & 0,3 & 0,3 \\
\hline$F_{t}$ & 43 & 3,29 & 36,31 & 0,4 & 0,3 & 0,2 & 0,435 & 0,851 & 5,4 & 4,0 & 2,7 \\
\hline \multicolumn{12}{|c|}{ Ciprés (Cupresssus lusitanica) } \\
\hline$F_{b}$ & 57,6 & 1,85 & 48,64 & 0,7 & 0,55 & 0,4 & 0,435 & 0,851 & 12,6 & 9,9 & 7,2 \\
\hline$E$ & 7600 & 2720 & 7600 & 1,0 & 0,9 & 0,8 & 1,064 & 0,905 & 7317 & 6585 & 5853 \\
\hline$E_{\min }$ & 7600 & 2720 & 2818 & 1,0 & 0,9 & 0,8 & 1,064 & 0,905 & 2713 & 2442 & 2171 \\
\hline$F_{c}$ & 14,3 & 1,56 & 11,73 & 0,7 & 0,55 & 0,4 & 0,476 & 0,696 & 2,7 & 2,1 & 1,6 \\
\hline $\mathrm{FC}_{\perp}$ & 24,1 & 1,65 & 20,35 & 1,0 & 1,0 & 1,0 & 0,599 & 1,000 & 12,2 & 12,2 & 12,2 \\
\hline $\mathrm{F}_{\mathrm{v}}$ & 9,4 & 1,54 & 6,47 & 0,5 & 0,5 & 0,5 & 0,435 & 0,920 & 1,3 & 1,3 & 1,3 \\
\hline$F_{t}$ & 52,2 & 3,87 & 44,08 & 0,4 & 0,3 & 0,2 & 0,435 & 0,851 & 6,5 & 4,9 & 3,3 \\
\hline \multicolumn{12}{|c|}{ Melina (Gmelina arborea) } \\
\hline$F_{b}$ & 50,3 & 0,87 & 42,47 & 0,7 & 0,55 & 0,4 & 0,435 & 0,851 & 11,0 & 8,6 & 6,3 \\
\hline$E$ & 8300 & 1490 & 8300 & 1,0 & 0,9 & 0,8 & 1,064 & 0,905 & 7991 & 7192 & 6393 \\
\hline $\mathrm{E}_{\min }$ & 8300 & 1490 & 5438 & 1,0 & 0,9 & 0,8 & 1,064 & 0,905 & 5235 & 4712 & 4188 \\
\hline$F_{c}$ & 31,8 & 5,86 & 20,52 & 0,7 & 0,55 & 0,4 & 0,476 & 0,696 & 4,8 & 3,7 & 2,7 \\
\hline $\mathrm{FC}_{\perp}$ & - & - & & 1,0 & 1,0 & 1,0 & 0,599 & 1,000 & - & - & - \\
\hline$F_{v}$ & 6,3 & 1,0 & 4,41 & 0,5 & 0,5 & 0,5 & 0,435 & 0,920 & 0,9 & 0,9 & 0,9 \\
\hline$F_{t}$ & 47,0 & 8,0 & 31,72 & 0,4 & 0,3 & 0,2 & 0,435 & 0,851 & 4,7 & 3,5 & 2,3 \\
\hline \multicolumn{12}{|c|}{ Roble coral (Terminalia amazonia) } \\
\hline$F_{b}$ & 74,6 & 1,58 & 62,99 & 0,7 & 0,55 & 0,4 & 0,435 & 0,851 & 16,3 & 12,8 & 9,3 \\
\hline$E$ & 11910 & 900 & 11910 & 1,0 & 0,9 & 0,8 & 1,064 & 0,905 & 11466 & 10320 & 9173 \\
\hline$E_{\min }$ & 11910 & 900 & 10057 & 1,0 & 0,9 & 0,8 & 1,064 & 0,905 & 9682 & 8714 & 7746 \\
\hline $\mathrm{F}_{\mathrm{c}}$ & 17,9 & 1,2 & 15,12 & 0,7 & 0,55 & 0,4 & 0,476 & 0,696 & 3,5 & 2,8 & 2,0 \\
\hline $\mathrm{FC}_{\perp}$ & 22,3 & 2,23 & 18,83 & 1,0 & 1,0 & 1,0 & 0,599 & 1,000 & - & - & - \\
\hline
\end{tabular}




\begin{tabular}{|c|c|c|c|c|c|c|c|c|c|c|c|}
\hline Propiedad & $\begin{array}{l}\text { Promedio } \\
(\mathrm{MPa})\end{array}$ & $\begin{array}{l}\text { Desviación } \\
\text { Estándar } \\
(\mathrm{MPa})\end{array}$ & $\begin{array}{c}\text { Quinto } \\
\text { Percentil } \\
\text { (MPa) }\end{array}$ & $\begin{array}{c}\text { Razón de } \\
\text { Resistencia } \\
\text { G1 }\end{array}$ & $\begin{array}{l}\text { Razón de } \\
\text { Resistencia } \\
\text { G2 }\end{array}$ & $\begin{array}{l}\text { Razón de } \\
\text { Resistencia } \\
\text { G3 }\end{array}$ & $\begin{array}{c}\text { Factor } \\
\text { de ajuste }\end{array}$ & $\begin{array}{l}\text { Ajuste } \\
\text { por } \\
\text { secado }\end{array}$ & Grado 1 & Grado 2 & Grado 3 \\
\hline $\mathrm{F}_{\mathrm{v}}$ & 4,5 & 2,57 & 0,85 & 0,5 & 0,5 & 0,5 & 0,435 & 0,920 & 0,2 & 0,2 & 0,2 \\
\hline$F_{t}$ & 56,7 & 2,72 & 47,88 & 0,4 & 0,3 & 0,2 & 0,435 & 0,851 & 7,1 & 5,3 & 3,5 \\
\hline \multicolumn{12}{|c|}{ Sura (Terminalia oblonga) } \\
\hline $\mathrm{F}_{\mathrm{b}}$ & 82,3 & 0,88 & 69,50 & 0,7 & 0,55 & 0,4 & 0,435 & 0,851 & 18,0 & 14,1 & 10,3 \\
\hline$E$ & 8070 & 1370 & 8070 & 1,0 & 0,9 & 0,8 & 1,064 & 0,905 & 7769 & 6992 & 6215 \\
\hline$E_{\min }$ & 8070 & 1370 & 5454 & 1,0 & 0,9 & 0,8 & 1,064 & 0,905 & 5251 & 4726 & 4201 \\
\hline$F_{c}$ & 37,5 & 0,843 & 31,67 & 0,7 & 0,55 & 0,4 & 0,476 & 0,696 & 7,3 & 5,8 & 4,2 \\
\hline $\mathrm{FC}_{\perp}$ & 35,3 & 1,16 & 29,81 & 1,0 & 1,0 & 1,0 & 0,599 & 1,000 & 17,8 & 17,8 & 17,8 \\
\hline$F_{v}$ & 7,6 & 0,78 & 6,36 & 0,5 & 0,5 & 0,5 & 0,435 & 0,920 & 1,3 & 1,3 & 1,3 \\
\hline$F_{t}$ & 65,1 & 2,85 & 54,97 & 0,4 & 0,3 & 0,2 & 0,435 & 0,851 & 8,1 & 6,1 & 4,1 \\
\hline \multicolumn{12}{|c|}{ Teca (Tectona grandis) } \\
\hline $\mathrm{F}_{\mathrm{b}}$ & 95,1 & 8,17 & 80,30 & 0,7 & 0,55 & 0,4 & 0,435 & 0,851 & 20,8 & 16,3 & 11,9 \\
\hline$E$ & 13100 & 1120 & 13100 & 1,0 & 0,9 & 0,8 & 1,064 & 0,905 & 12612 & 11351 & 10090 \\
\hline$E_{\min }$ & 13100 & 1120 & 11062 & 1,0 & 0,9 & 0,8 & 1,064 & 0,905 & 10650 & 9585 & 8520 \\
\hline$F_{c}$ & 42,5 & 4,91 & 34,15 & 0,7 & 0,55 & 0,4 & 0,476 & 0,696 & 7,9 & 6,2 & 4,5 \\
\hline $\mathrm{FC}_{\perp}$ & 9,6 & 1,05 & 7,87 & 1,0 & 1,0 & 1,0 & 0,599 & 1,000 & 4,7 & 4,7 & 4,7 \\
\hline$F_{v}$ & 6,2 & 1,05 & 4,20 & 0,5 & 0,5 & 0,5 & 0,435 & 0,920 & 0,8 & 0,8 & 0,8 \\
\hline$F_{t}$ & 91,2 & 20,1 & 52,45 & 0,4 & 0,3 & 0,2 & 0,435 & 0,851 & 7,8 & 5,8 & 3,9 \\
\hline \multicolumn{12}{|c|}{ Chancho blanco (Vochysia guatemalensis) } \\
\hline$F_{b}$ & 40,4 & 1,03 & 34,11 & 0,7 & 0,55 & 0,4 & 0,435 & 0,851 & 8,8 & 6,9 & 5,0 \\
\hline$E$ & 6410 & 1070 & 6410 & 1,0 & 0,9 & 0,8 & 1,064 & 0,905 & 6171 & 5554 & 4937 \\
\hline$E_{\min }$ & 6410 & 1070 & 4372 & 1,0 & 0,9 & 0,8 & 1,064 & 0,905 & 4209 & 3788 & 3367 \\
\hline$F_{c}$ & 21,3 & 1,31 & 17,99 & 0,7 & 0,55 & 0,4 & 0,476 & 0,696 & 4,2 & 3,3 & 2,4 \\
\hline $\mathrm{FC}_{\perp}$ & 14,5 & 1,58 & 11,90 & 1,0 & 1,0 & 1,0 & 0,599 & 1,000 & 7,0 & 7,0 & 7,0 \\
\hline$F_{v}$ & 6,3 & 1,64 & 3,19 & 0,5 & 0,5 & 0,5 & 0,435 & 0,920 & 0,6 & 0,6 & 0,6 \\
\hline$F_{t}$ & 39,4 & 2,77 & 33,27 & 0,4 & 0,3 & 0,2 & 0,435 & 0,851 & 4,9 & 3,7 & 2,5 \\
\hline
\end{tabular}

Donde: $\mathrm{F}_{\mathrm{b}}$ : Esfuerzo en flexión, E: Módulo de elasticidad, $\mathrm{E}_{\min }$ : Módulo de elasticidad mínimo, $\mathrm{F}_{\mathrm{c}}$ : Esfuerzo en compresión paralela, $\mathrm{F}_{\mathrm{c} \perp}$ : Esfuerzo en Compresión perpendicular al grano, $F_{\mathrm{v}}$ : Esfuerzo en cortante, $\mathrm{F}_{\mathrm{t}}$ : Esfuerzo en tensión paralela al grano. 